\title{
Partitioning the Effects of Eco-Evolutionary Feedbacks on Community Stability ${ }^{\star}$
}

\author{
Swati Patel, ${ }^{1, \dagger}$ Michael H. Cortez, ${ }^{2}$ and Sebastian J. Schreiber ${ }^{3}$ \\ 1. Graduate Group in Applied Mathematics, University of California, Davis, California 95616; Faculty of Mathematics, University of Vienna, \\ 1090 Vienna, Austria; Department of Mathematics, Tulane University, New Orleans, Louisiana 70115; 2. Department of Mathematics and \\ Statistics, Utah State University, Logan, Utah 84322; 3. Department of Evolution and Ecology and Center for Population Biology, \\ University of California, Davis, California 95616
}

Submitted April 29, 2017; Accepted September 22, 2017; Electronically published January 24, 2018

Online enhancements: appendixes.

\begin{abstract}
Aвstract: A fundamental challenge in ecology continues to be identifying mechanisms that stabilize community dynamics. By altering the interactions within a community, eco-evolutionary feedbacks may play a role in community stability. Indeed, recent empirical and theoretical studies demonstrate that these feedbacks can stabilize or destabilize communities and, moreover, that this sometimes depends on the relative rate of ecological to evolutionary processes. So far, theory on how eco-evolutionary feedbacks impact stability exists only for a few special cases. In our work, we develop a general theory for determining the effects of eco-evolutionary feedbacks on stability in communities with an arbitrary number of interacting species and evolving traits for when evolution is slow and fast. We characterize how eco-evolutionary feedbacks lead to stable communities that would otherwise be unstable, and vice versa. Additionally, we show how one can identify the roles of direct and indirect feedbacks between ecological and evolutionary processes on stability and how the effects of those feedbacks depend on the rate of evolution relative to the ecological timescales. Applying our methods to models of competing species and food chains, we demonstrate how the functional form of trade-offs, genetic correlations between traits, and the rate of evolution determine whether eco-evolutionary feedbacks stabilize or destabilize communities.
\end{abstract}

Keywords: stability, community dynamics, indirect effects, competition, food chain, multivariate selection.

\section{Introduction}

In recent years, there has been growing empirical evidence highlighting that eco-evolutionary feedbacks can dramati-

\footnotetext{
* Originally presented as part of the American Society of Naturalists symposium "Evolution in a Community Context," organized by Peter C. Zee and coorganized and moderated by Casey P. terHorst, at the meeting of the Ecological Society of America in Fort Lauderdale, Florida, in August 2016.

$\dagger$ Corresponding author; e-mail: swpatel@ucdavis.edu.

ORCIDs: Patel, http://orcid.org/0000-0003-3502-6895; Schreiber, http://orcid .org/0000-0002-5481-4822.
}

Am. Nat. 2018. Vol. 191, pp. 381-394. (C) 2018 by The University of Chicago. 0003-0147/2018/19103-57695\$15.00. All rights reserved.

DOI: $10.1086 / 695834$ cally impact community-level properties, including stability (Pimentel et al. 1963; Yoshida et al. 2003; Becks et al. 2010; Sanchez and Gore 2013; Steiner and Masse 2013; Kasada et al. 2014). Moreover, there is evidence that evolution happens at various rates, ranging from slower than to commensurate to ecological rates (Darimont et al. 2009; terHorst 2010; DeLong et al. 2016; Hendry 2016), and this affects the impact of feedbacks (Becks et al. 2010; Turcotte et al. 2011; Reznick 2013). Theoretical work to mechanistically understand how ecoevolutionary feedbacks impact stability has mostly focused on specific ecological modules with just one or two evolving species (Abrams and Matsuda 1997; Fussmann et al. 2007; Cortez and Ellner 2010; Vasseur et al. 2011; Hendry 2013; Cortez 2016). Here, we present a general framework to determine the role of eco-evolutionary feedbacks on stability, accounting for the relative timescales of ecological and evolutionary processes.

Determining when an ecosystem of interacting species is stable is of fundamental importance in ecology, offering insight into how the ecosystem will respond to inevitable perturbations. A stable system is able to withstand small but frequent perturbations (Schreiber 2006). Understanding how eco-evolutionary feedbacks impact stability is even more important given the rise of anthropogenic changes, including climate change and habitat disturbances, which fundamentally perturb ecosystems. These perturbations are likely to lead not only to ecological responses - that is, population density changes - but also to drive an evolutionary response - that is, trait changes in the populations - due to changing selection pressures (Darimont et al. 2009; Hendry et al. 2011; Lankau et al. 2011; Alberti 2015). Indeed, in a meta-analysis, Darimont et al. (2009) showed that trait change was more rapid in human-disturbed ecosystems than from natural causes. As ecosystems are perturbed, evolutionary trait changes in the enclosed populations and subsequent feedbacks with the ecological dynamics can play a critical role in determining how ecosystems respond to perturbations. 
Previous work has explored how eco-evolutionary feedbacks affect community stability, but this work has been limited to either small, specific communities with one or two evolving species or numerical rather than analytical results. In particular, analyses of predator-prey (Abrams and Matsuda 1997; Cortez and Ellner 2010; Cortez 2016), two-species competition (Vasseur et al. 2011), three-species apparent competition (Schreiber et al. 2011; Schreiber and Patel 2015), and three-species intraguild predation (Patel and Schreiber 2015) models highlight that the stabilities of eco-evolutionary coupled systems depend on the relative timescales of the ecological and evolutionary process. Theoretical studies such as these are important given the empirical evidence that evolution can happen at varying timescales relative to ecological processes (DeLong et al. 2016). In more complex communities, the increased number of direct and indirect interactions make understanding the impacts of eco-evolutionary feedbacks on stability more challenging (Hendry 2016), and thus far, studies have predominantly used numerical techniques (Kondoh 2003; Barabás and D’Andrea 2016), which can sometimes limit their ability to provide a mechanistic understanding. Hence, a general analytical theory of when and how ecoevolutionary feedbacks alter stability in general ecological communities is lacking.

In this article, we address this challenge through a new approach for determining stability in models that couple ecological and evolutionary dynamics. In particular, we present analytical conditions to infer stability for slow and fast evolution in a general model for coupled eco-evolutionary dynamics and describe the role eco-evolutionary feedbacks have on these conditions. Specifically, we are able to partition the effects of ecology (direct effects of density changes on population dynamics), evolution (direct effects of trait changes on selection), and eco-evolutionary feedbacks (indirect effects of density changes on population dynamics mediated by trait changes and indirect effects of trait changes on selection mediated by population density changes) on stability, enabling a more mechanistic understanding of stability in communities with ecological and evolutionary dynamics. Importantly, we show that these feedbacks can fundamentally change predictions on the qualitative dynamics compared to predictions when ecology and evolution are uncoupled. To demonstrate the utility of our approach, we apply it to models of two competing species with one species evolving in one trait and a food chain with one species evolving in multiple traits.

\section{Model}

To evaluate the effects of eco-evolutionary feedbacks on stability, we examine a general multispecies model that couples population dynamics with evolutionary trait dynamics. We consider $k$ species interacting, with population densities
$N_{1} \ldots N_{k}$, and $\ell$ ecologically important evolving traits, with trait values $x_{1} \ldots x_{\ell}$. The model is

$$
\begin{aligned}
\frac{d N_{i}}{d t} & =N_{i} f_{i}(N, x), \\
\frac{d x_{j}}{d t} & =\varepsilon g_{j}(N, x),
\end{aligned}
$$

where $N=\left(N_{1} \ldots N_{k}\right)$ and $x=\left(x_{1} \ldots x_{\ell}\right)$. The function $f_{i}$ represents the per capita fitness of species $i$ and describes how the growth of species $i$ depends on the population densities of all species and all traits. The function $g_{j}$ represents the selection function and describes how trait $j$ evolves due to the selective pressures imposed by all population densities and traits.

Finally, $\varepsilon$ determines the timescale separation between the ecological and evolutionary dynamics. When $\varepsilon$ is small, the evolutionary process occurs very slowly relative to the ecological processes (hereafter, "slow evolution"). For example, slow evolution occurs when there is low genetic variance, which constrains evolutionary potential as long as the fitness gradient is not simultaneously large. When $\varepsilon$ is large, the evolutionary process occurs very quickly relative to the ecological processes (hereafter, "fast evolution"). For example, fast evolution, relative to ecology, occurs when species densely occupy an environment, which constrains their growth, but there is high population turnover. Hence, population densities do not change much over many generations, while traits may change. Indeed, many classical population genetics models of evolution and coevolution assume an extreme version of this scenario in which population densities remain constant while genotypic frequencies change (Lande 1976; Seger 1988; Gavrilets 1997; Nuismer and Doebeli 2004). The limit of fast evolution also serves as a useful heuristic tool for understanding ecoevolutionary dynamics when the timescale separation is less extreme or absent (Cortez and Ellner 2010; Cortez and Weitz 2014; Cortez 2015). Notably, this model form has the flexibility to incorporate any number and combination of species interactions, including predator-prey, mutualistic, and competitive interactions. In particular, we make no initial assumptions about the functional form of growth rates $f_{i}$, which determine the dynamical consequences of the species interactions. This model form also has the flexibility to incorporate any number of evolving traits, including coevolution between multiple species and multitrait evolution within one species. Our modeling of the evolutionary dynamics implicitly assumes that (1) for each trait, evolution can be represented by changes in a single continuous quantity (e.g., the mean of a quantitative trait or the frequency of an allele) and that (2) all changes in these continuous quantities are attributed to evolutionary selection.

These assumptions are met by a number of eco-evolutionary modeling frameworks, such as adaptive dynamics (Marrow 
et al. 1996; Geritz et al. 1998) and quantitative genetic approaches based on Lande (1976). To demonstrate the flexibility of our framework, we describe two particular applications of the Lande approach. For example, when one normally distributed quantitative trait is evolving in each species, with constant genetic variances, and selection is frequency independent, the evolutionary dynamics are given by

$$
\frac{d x_{i}}{d t}=\varepsilon g_{i}=v_{i} \frac{\partial f_{i}}{\partial x_{i}} \quad i=1 \ldots k
$$

where $x_{i}$ and $v_{i}$ are the means and genetic variances of the evolving traits, respectively, and $f_{i}$ is the mean fitness. Note that the traits change in the direction of increasing fitness due to the selection gradients. When all the genetic variances and the selection gradient are sufficiently small relative to growth when measured on the same scale, evolution is slow, and this generates a natural timescale separation between the ecological and evolutionary processes.

Alternatively, when one species, say species $i$, is evolving in multiple quantitative traits, the evolutionary dynamics are given by

$$
\frac{d x_{j}}{d t}=\varepsilon g_{j}=\sum_{m=1}^{\ell} v_{m j} \frac{\partial f_{i}}{\partial x_{m}} \quad j=1 \ldots \ell,
$$

where $x_{j}$ is the mean of the $j$ th evolving trait and $v_{m j}$ is the genetic covariance between traits $m$ and $j$. Here, selection acting on one trait affects the evolutionary dynamics of any other covarying trait and, hence, may affect the eco-evolutionary dynamics (Lande and Arnold 1983; Kopp and Matuszewski 2013).

\section{Equilibrium Stability and Eco-Evo Feedbacks}

How a system at equilibrium responds to perturbations determines its stability. In general, an equilibrium is stable if the system returns to the equilibrium following small density or trait perturbations. Equilibrium stability is determined by the eigenvalues of the Jacobian matrix. In particular, for differential equation models, if the stability modulus - that is, the largest real part of the eigenvalues - of the Jacobian is negative (positive), then the equilibrium is stable (unstable). For any matrix, $M$, we use $s(M)$ to denote the stability modulus.

The Jacobian for (1) has the form

$$
J=\left[\begin{array}{cc}
A & B \\
\varepsilon C & \varepsilon D
\end{array}\right]
$$

where $A, B, C$, and $D$ are submatrices and $\varepsilon$ is as in (1). These submatrices represent different components of the eco- evolutionary dynamics of the community. Submatrix $A$ captures the direct effects ecological processes have on species densities (fig. 1I); it has elements $\partial\left(N_{i} f_{i}\right) / \partial N_{k}$, that is, how the growth rate of species $i$ changes with the density of species $k$. Commonly referred to as the community interaction matrix (Levins 1968; May 1973; Pimm and Lawton 1978; Bender et al. 1984; Yodzis 1988), it is the Jacobian of the ecological dynamics and determines stability in the absence of evolutionary dynamics. We call the system ecologically stable if $s(A)<0$; when traits are fixed, population densities return to equilibrium following a perturbation. Analogously, $D$ captures the direct effects of evolutionary processes on trait dynamics and has elements $\partial g_{j} / \partial x_{\ell}$, that is, how selection on trait $j$ changes with trait $\ell$. We call the system evolutionarily stable if $s(D)<0$; when population densities are fixed, traits return to equilibrium following a perturbation. Submatrix $B$ captures the effects of evolution on ecology; it has elements $\partial\left(N_{i} f_{i}\right) / \partial x_{\ell}$, that is, how the growth rate of species $i$ changes with trait $\ell$. Submatrix $C$ captures the effects of ecology on evolution; it has elements $\partial g_{j} / \partial N_{k}$, that is, how selection on trait $j$ changes with the density of species $k$.

The equilibrium of model (1) is overall stable if $s(J)<0$ and overall unstable if $s(J)>0$. When ecological and evolutionary dynamics are uncoupled, $B$ and $C$ are zero matrices (implying no effects of ecology on evolution, and vice versa) and only the direct effects captured in $A$ and $D$ determine stability. In this case, $s(J)$ is the maximum of $s(A)$ and $s(D)$. Hence, the stability of the whole system is determined by the stabilities of the separate ecological and evolutionary components. An equilibrium must be both ecologically stable and evolutionarily stable to be overall stable (i.e., $s(J)<$ 0 if and only if $s(A)<0$ and $s(D)<0)$.

On the other hand, when ecological and evolutionary dynamics are coupled, indirect eco-evolutionary feedbacks between population densities and traits also play a role in overall stability. Coupling of ecological and evolutionary processes yields two indirect feedback loops that are important for overall stability when evolution is slow or fast: (1) the evo-eco-evo feedback and (2) the eco-evo-eco feedback. In the evo-ecoevo feedback, changes in traits cause changes in population densities, which, in turn, alter selection on the traits (fig. 1II). Mathematically, the three steps are captured by $C A^{-1}(-B)$. In the first step of the evo-eco-evo feedback, a change in the traits drives changes in population growth, given by $B$. In the second step, the population densities respond to this change in growth and reach a new equilibrium, given by $A^{-1}(-B)$. This expression was used by Levins (1968), Bender et al. (1984), and Yodzis (1988) to describe how population densities in communities respond to external perturbations $(\Delta N)$. In the final step, changes in population densities drive changes in selection pressures, given by $C$, closing the feedback loop. Similarly, in the eco-evo-eco feedback, changes in population densities cause changes in the traits, which in turn alter pop- 

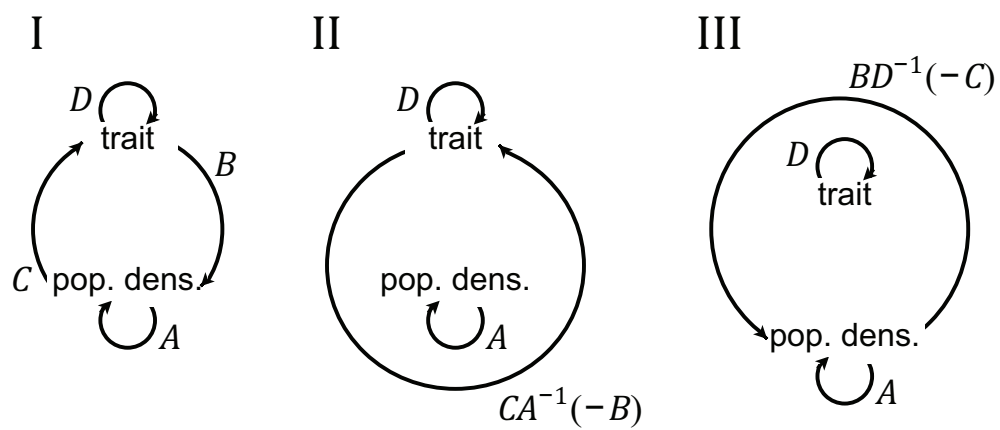

Figure 1: Schematics showing important feedbacks in coupled eco-evolutionary systems. Traits and population densities have direct effects (clockwise arrows) on themselves and on each other (I). These generate the indirect evo-eco-evo feedback (counterclockwise arrow in $I I$ ) and the indirect eco-evo-eco feedback (counterclockwise arrow in III). If there is a timescale separation between the ecological and evolutionary dynamics, $I I$ and III show the critical feedbacks for determining stability when evolution is slow and fast, respectively.

ulation dynamics (fig. 1III). The corresponding three steps are captured by the matrix $B D^{-1}(-C)$.

\section{General Stability Results for Slow and Fast Evolution}

When and how do these direct and indirect feedbacks affect the stability of equilibria when ecology and evolution are coupled? To address this question, we discuss the stability conditions for cases when there is a timescale separation between ecological and evolutionary processes. The timescale separation enables us to derive analytical conditions that partition the effects of ecology, evolution, and eco-evolutionary feedbacks on stability. Mainly, we show that the evo-eco-evo feedback (fig. $1 I I$ ) is critical for stability when evolution is slow and that the eco-evo-eco feedback (fig. 1III) is critical for stability when evolution is fast. We begin with the case of a single slowly evolving trait coupled to multispecies population dynamics to present and describe the intuition behind the results.

\section{Communities with a Single Slowly Evolving Trait}

We consider a community with $k$ interacting species, in which one focal species is evolving in a single trait. Throughout this section, we assume that the evolutionary process is slow relative to the ecological processes ( $\varepsilon$ is small). In this case, the Jacobian for an equilibrium point can be represented by

$$
J=\left[\begin{array}{cc}
A & b \\
\varepsilon c & \varepsilon d
\end{array}\right],
$$

where $b$ and $c$ are column and row vectors, respectively, with length equal to the number of species, and $d$ is a scalar. Then, the eco-evolutionary equilibrium is stable if the equilibrium is ecologically stable (i.e., $s(A)<0$ ) and

$$
d+c A^{-1}(-b)<0 .
$$

In words, stability requires that the sum of the direct evolutionary feedback and the indirect evo-eco-evo feedback is negative. Hence, if the feedback is negative $\left(c A^{-1}(-b)\right)$, then the more negative it is, the more it has a stabilizing effect. Alternatively, if the feedback is positive, then the more positive it is, the more it has a destabilizing effect. A proof of this condition is given in appendix A (apps. A-E are available online).

Intuitively, the stability conditions describe a response to perturbation that proceeds in two steps: a fast ecological response, which leads to the condition $s(A)<0$, and a slow evolutionary response, which leads to condition (6). In the fast response, the population densities either quickly converge to a population equilibrium (ecological stability) or diverge from it (ecological instability). Hence, stability in the fast (ecological) dynamic is always necessary.

In the slow response, the trait slowly changes, due to the direct evolutionary effects. These slow trait changes drive the population densities to continuously respond and quickly approach a new equilibrium corresponding to the changed trait. This, in turn, affects the selection pressures, giving rise to the indirect evo-eco-evo feedback. When condition (6) is met, these direct and indirect effects act in conjunction to drive the traits and population densities to return to the ecoevolutionary equilibrium (i.e., overall stability; fig. 2A).

From this condition, an equilibrium can be characterized by one of four possible cases, differentiated by whether it is evolutionarily stable as well as whether it is overall stable in the coupled system. Specifically, an equilibrium can be (i) evolutionarily stable and overall stable, (ii) evolutionarily stable and overall unstable, (iii) evolutionarily unstable and overall unstable, or (iv) evolutionarily unstable and overall stable (see fig. 2). Interestingly, scenario ii highlights that an equilibrium that is ecologically stable and evolutionarily stable need not be overall stable; this occurs when the indirect evo-eco-evo feedback is sufficiently destabilizing. Conversely, scenario iv highlights that even if an equilibrium is evolutionarily unstable, it can be stabilized when the evo-eco-evo feedback is sufficiently stabilizing to counter the destabilizing effect of the 

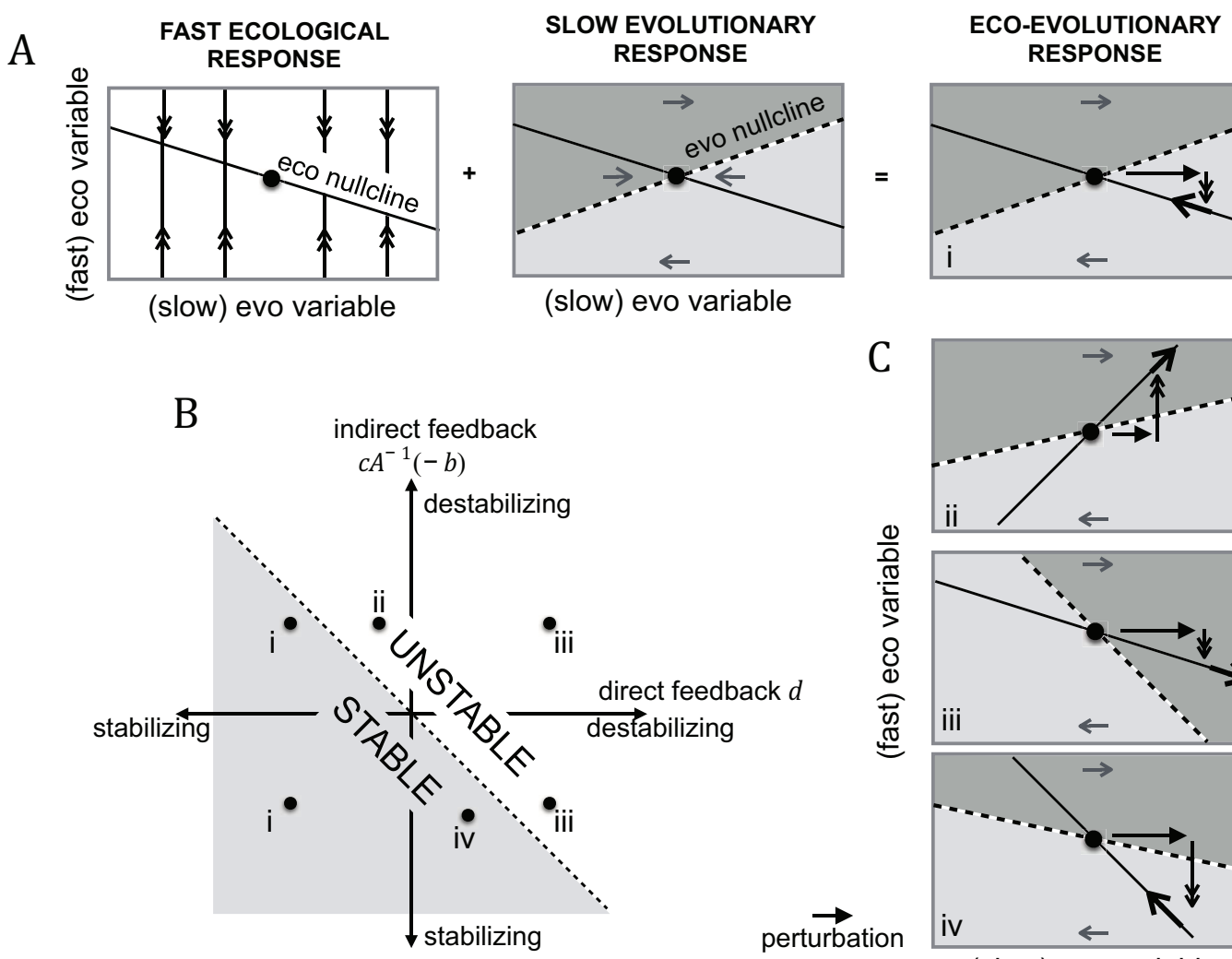

direct feedback $d$ $\overrightarrow{\text { estabilizing }}$

\section{C}
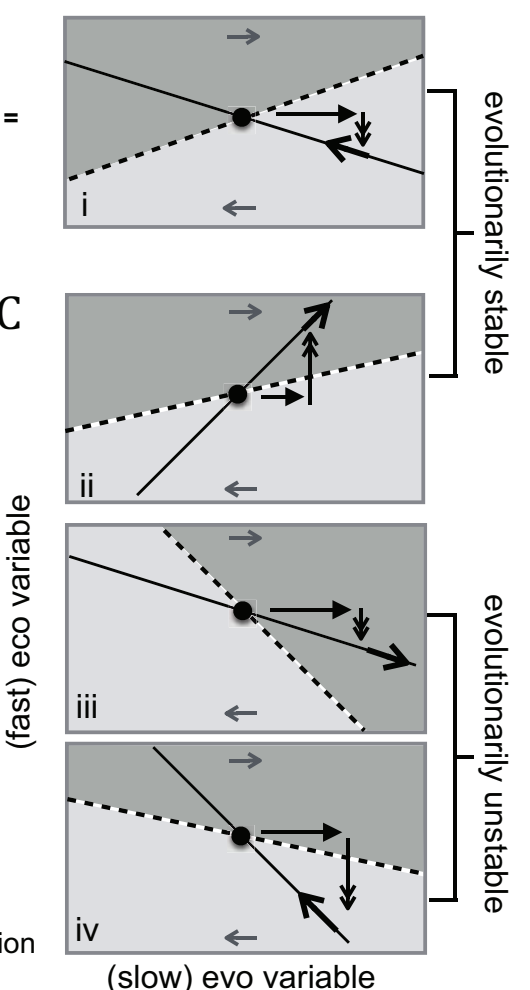

Figure 2: Visual representation of the stability results for a community with a single slowly evolving trait. In $A$, the three schematic plots show the fast ecological dynamics (double black arrows in first plot), the slow evolutionary dynamics (gray arrows in second plot), and their joint response (black diagonal arrow in third plot) to a perturbation (horizontal black arrow in third plot). In $B$, the dotted line depicts when the sum of the direct $(d)$ and the indirect evo-eco-evo feedback effect $\left(c A^{-1}(-b)\right)$ is zero, which separates stable from unstable. The points correspond to the four possible scenarios discussed in the text: evolutionarily stable and overall stable (i), evolutionarily stable and overall unstable (ii), evolutionarily unstable and overall unstable (iii), and evolutionarily unstable but overall stable (iv). The two-step eco-evolutionary responses for each of these scenarios are shown in the schematic plots in $A$ and $C$.

direct evolutionary feedbacks. In this latter case, we say that the evo-eco-evo feedback stabilizes the equilibrium.

\section{Communities with Multiple Slowly Evolving Traits}

Suppose now we wish to model a community in which there are multiple evolving traits. This is appropriate for when many species are each evolving in a single trait (as in eq. [2]), a single species is evolving in multiple traits (as in eq. [3]), or most generally, when many species are evolving in multiple traits. When there are multiple evolving traits, collectively in all the species, the response to perturbations and the conditions for equilibrium stability are nearly identical to the single-trait case. In particular, the eco-evolutionary equilibrium is stable if the equilibrium is ecologically stable (i.e., $s(A)<0$ ) and

$$
s\left(D+C A^{-1}(-B)\right)<0 .
$$

For details, see appendix A. Analogous to the previous section, the fast ecological response leads to condition $s(A)<0$, and the slow evolutionary response leads to the condition (7) for stability. The key difference between the multiple-trait and single-trait cases is that when there are multiple traits, the direct evolutionary feedback $(D)$ and the indirect evoeco-evo feedback are represented by matrices $\left(C A^{-1}(-B)\right)$. Hence, stability is determined by the stability modulus of this matrix sum. As with a single evolving trait, an equilibrium that is ecologically stable and evolutionarily stable can be unstable due to the evo-eco-evo feedbacks. On the other hand, evo-eco-evo feedbacks can stabilize an equilibrium, even if it is evolutionarily unstable.

\section{Communities with Multiple Fast-Evolving Traits}

To understand the effects of eco-evolutionary feedbacks when evolution is fast, we study the opposite limit (large $\varepsilon$ ). For example, this may occur when population densities are constrained near a carrying capacity and their net growth is limited, but there is high population turnover, enabling evolu- 
tion to still occur. The eco-evolutionary equilibrium is stable if the equilibrium is evolutionarily stable (i.e., $s(D)<0$ ) and

$$
s\left(A+B D^{-1}(-C)\right)<0 .
$$

Equation (8) shows that when evolution is fast, equilibrium stability requires that the direct ecological effects $(A)$ in addition to the indirect eco-evo-eco feedbacks $\left(B D^{-1}(C)\right)$ are negative. Note that when the ecological variables are only onedimensional, this condition simplifies to $a+b D^{-1}(-c)<0$ (analogous to the case of a single slowly evolving trait).

When evolutionary processes are fast relative to ecological processes, the two-step response to perturbations is reversed: the evolutionary response is fast, which leads to the condition $s(D)<0$, and the ecological response is slow, which leads to condition (8). In the fast response, provided the equilibrium is evolutionarily stable, traits quickly approach the trait equilibria, due to the direct evolutionary effects. In the slow response, the population densities slowly change, due to the direct ecological effects. These density changes drive the traits to continue to change, which in turn affects the growth of each population, giving rise to the indirect eco-evo-eco feedback. When condition (8) is met, these direct and indirect effects act in conjunction to drive the traits and population densities to return to the eco-evolutionary equilibrium.

\section{Applications to Two Competing Species}

Here, we apply our stability conditions to determine how ecoevolutionary feedbacks can alter stability between two competing species. These examples show how purely qualitative information (signs of effects) can be used to determine the role of eco-evolutionary feedbacks on stability. Classic twospecies competition theory asserts that a coexistence equilibrium is ecologically stable if each species competes more strongly intraspecifically than interspecifically (Tilman 2007). Conversely, if both species compete more strongly interspecifically than intraspecifically, then the equilibrium is unstable and the two species do not coexist; initial densities determine which species is competitively excluded (fig. 3 ).

We assume that one of the two competing species (species 1) has a quantitative trait that is subject to frequencyindependent selection. Under the Lande (1976) framework, this implies that a trait equilibrium occurs when the fitness gradient is zero (see eq. [2]) and is evolutionarily stable (unstable) when fitness is locally maximized (minimized; fig. 3). We examine two different types of traits and show that in one case, eco-evolutionary feedbacks are stabilizing for slow and fast evolution, while in another, they are destabilizing. Through these two examples, we show how purely qualitative information (signs of effects) can be used to determine the role of eco-evolutionary feedbacks on stability.
A

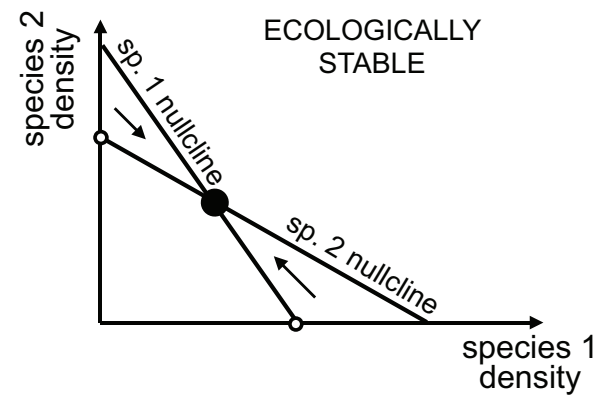

C

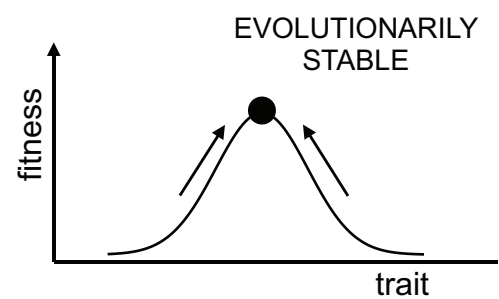

B
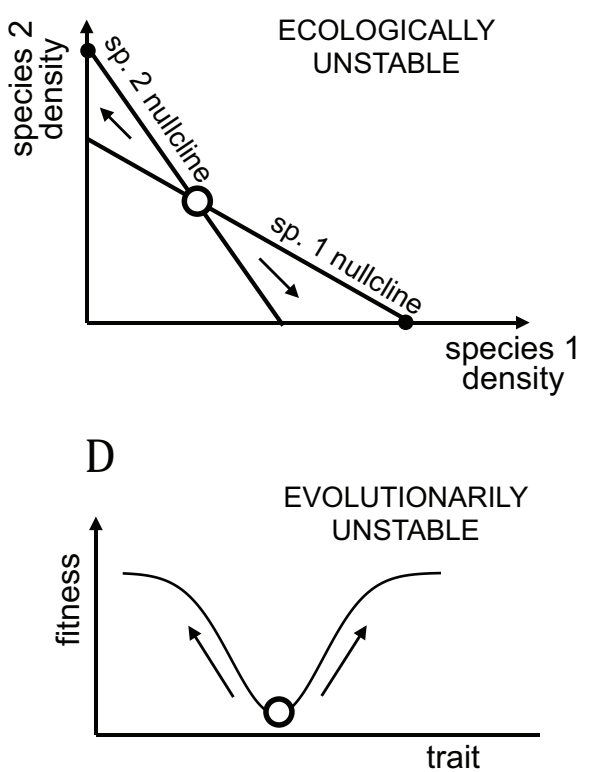

Figure 3: Schematics of stable and unstable equilibria in a classic two-competing-species ecological model ( $A, B)$ and an evolutionary model $(C, D)$. When intraspecific competition is greater than interspecific competition, the coexistence equilibrium between two competing species is stable $(A)$ and otherwise unstable $(B)$. With frequency-independent selection, equilibria at local fitness maxima are stable $(C)$ and equilibria at local fitness minima are unstable $(D)$. 


\section{Competition Model I: Eco-Evolutionary Feedbacks Are Stabilizing}

Both competing species experience intra- and interspecific competition, so that growth rates are negatively related to population densities. Therefore, the ecological Jacobian matrix is

$$
A=\left[\begin{array}{ll}
- & - \\
- & -
\end{array}\right],
$$

with the negative effects of intra- and interspecific competition captured in the signs of the diagonal and off-diagonal elements, respectively.

In the first model, we assume that the evolving trait in species 1 exhibits a trade-off in the ability of individuals to compete with conspecifics versus heterospecifics. Following Vasseur et al. (2011), this trait reduces the antagonistic effects of species 2 on species 1 , increases the antagonistic effects of species 1 on species 2 , and increases intraspecific antagonism (fig. 4I). Traits such as body size or aggressiveness fall into this category. For example, aggressiveness in social spiders (Anelosimus studious) leads to increased competitive ability against heterospecifics but a reduction in resource-use efficiency, increasing intraspecific competition (Pruitt and Riechert 2009).

This qualitative information about the type of trait determines the signs of $b$ and $c$ vectors in the Jacobian matrix. In particular, there are four important relationships between demography and selection that determine the effects of ecoevolutionary feedbacks on stability. First, increased trait values reduce the fitness of species 2 . Second, increased density of species 1 selects for decreasing the trait. Third, increased density of species 2 selects for increasing the trait. Finally, consistent with Vasseur et al. (2011), since selection is frequency independent, the fitness gradient equals zero at a trait equilibrium (Lande 1976; fig. 3). Altogether, these four statements determine the signs of vectors $b$ and $c$ in the Jacobian matrix at an equilibrium

$$
b=\left[\begin{array}{c}
0 \\
-
\end{array}\right], \quad c=\left[\begin{array}{ll}
- & +
\end{array}\right]
$$

When the evolutionary dynamics are slow relative to the ecological dynamics, the eco-evolutionary feedback can stabilize an evolutionarily unstable equilibrium. Indeed, the sign of the evo-eco-evo pathway is given by

$$
\begin{aligned}
& c A^{-1}(-b)=\left[\begin{array}{ll}
- & +
\end{array}\right]\left[\begin{array}{ll}
- & + \\
+ & -
\end{array}\right]\left[\begin{array}{l}
0 \\
+
\end{array}\right]=[-] . \\
& \Delta N=\left[\begin{array}{l}
+ \\
-
\end{array}\right]
\end{aligned}
$$

In the first step of this pathway, a small increase in the trait leads to an increase in the density of species 1 and decrease of species 2 (from $\Delta N$ ). These population density
I

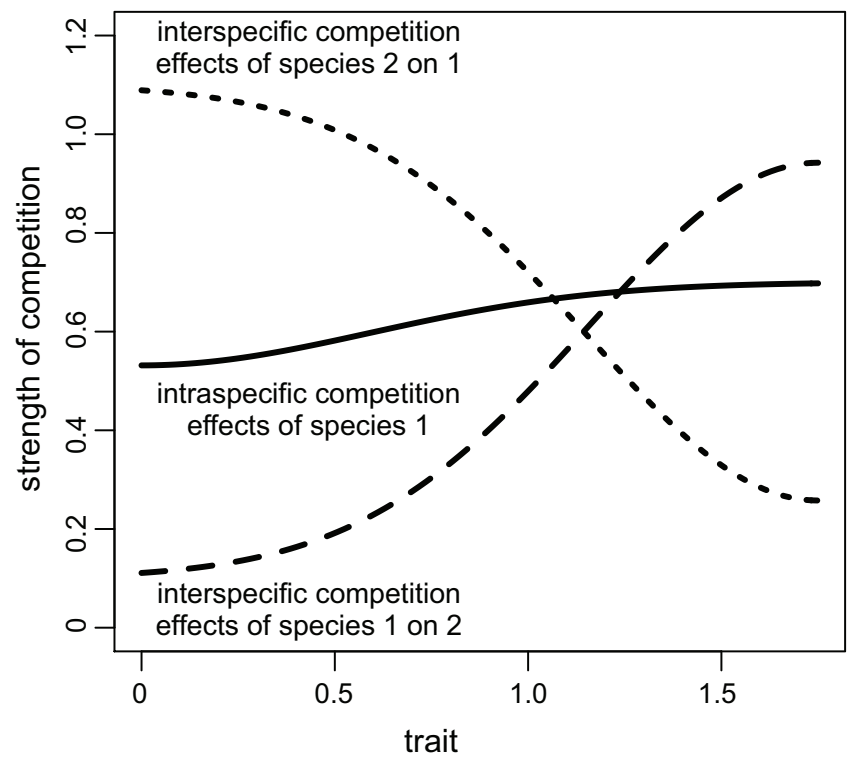

II

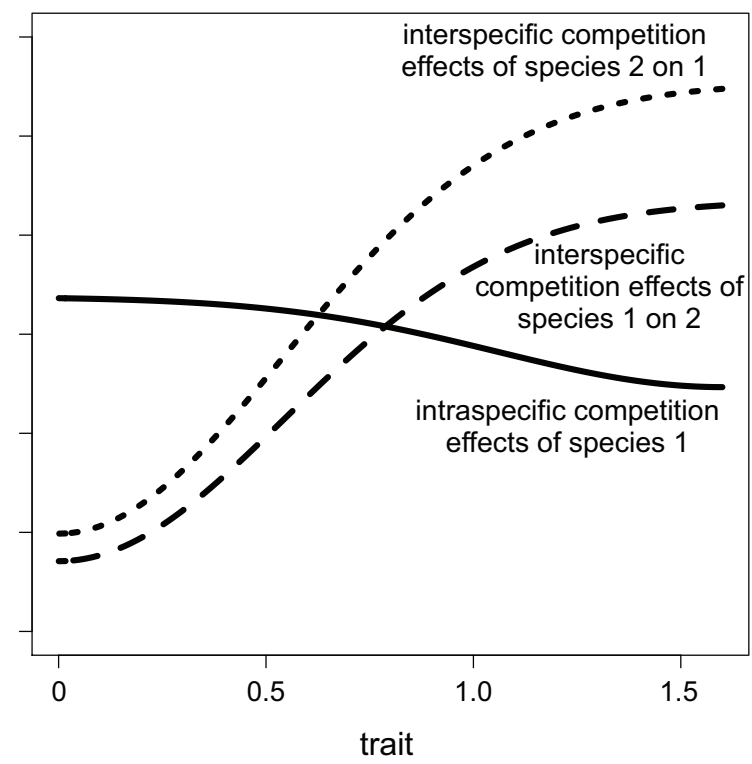

Figure 4: Intra- and interspecific competition for traits in competition models I and II. In model I, increasing the trait value increases intraspecific competition within species 1 as well as interspecific competition effects of species 1 on 2 while decreasing interspecific competition effects of species 2 on 1 . In model II, increasing the trait values increases interspecific competition effects between species 1 and 2 while decreasing intraspecific competition effects within species 1. For details of the models, see appendix C. 
changes result in a selective pressure for decreased trait values, which counteracts the perturbation. This creates a negative feedback, which has stabilizing effects (corresponding to the bottom half of fig. $2 B$ ) and can stabilize an equilibrium that is evolutionarily unstable $(d>0)$.

Similarly, when the evolutionary dynamics are fast relative to the ecological dynamics, the eco-evolutionary feedback can stabilize an ecologically unstable equilibrium. The stabilizing feedback comes from the eco-evo-eco pathway, given by

$$
b d^{-1}(-c)=\left[\begin{array}{cc}
0 & 0 \\
+ & -
\end{array}\right]
$$

Analogous to the community matrix of direct ecological effects, this $2 \times 2$ matrix represents the indirect effect population densities have on each other's growth via an indirect pathway. In general, it is difficult to determine the effects of adding matrices on the stability. However, for $2 \times 2$ matrices, the stability modulus is negative if the trace is negative and the determinant is positive. Adding the eco-evolutionary feedback term to matrix $A$ has two effects: it increases the product of the diagonal terms, and it decreases the product of the off-diagonal terms. This makes the determinant of $A+$ $b d^{-1}(-c)$ more positive and the trace more negative, which are both stabilizing effects.

To summarize, the eco-evo-eco and evo-eco-evo feedbacks are both stabilizing. But when is this sufficient to result in a stable equilibrium? To address this question, we compared our general theory to the specific two-species competition differential equation model from Vasseur et al. (2011). We note that our theory applies when evolution is slow or fast but, in general, does not determine stability at intermediate rates (see, however, app. B for a special exception). By incrementally varying the heritability, which determines the relative rate of evolution to ecological dynamics in the model, we examined stability from slow to fast evolution of four types of equilibria: (1) ecologically and evolutionarily stable, (2) ecologically stable and evolutionarily unstable, (3) ecologically unstable and evolutionarily stable, and (4) ecologically and evolutionarily unstable (for more details, see app. C; fig. 5).

We find that if an equilibrium is ecologically stable and evolutionarily unstable $(d>0)$, then the evo-eco-evo feedbacks are sufficient to stabilize this equilibrium when evolution is slow (top right in fig. 5A). For faster evolutionary rates, our results match the predictions from our theory that these equilibria will be unstable (fig. $5 B$ ). In figure $5 \mathrm{~A}$, we show the critical heritability at which this loss of stability occurs (color gradient at top right). An equilibrium that is more ecologically stable (smaller $s(A)$ ) has a larger critical heritability to lose stability than equilibria that are less ecologically stable (horizontal transects in fig. $5 \mathrm{~A}$, top right).

Analogously, we find that if an equilibrium is ecologically unstable and evolutionarily stable, then the eco-evo-eco feed- backs are sufficient to stabilize the equilibrium when evolution is fast (fig. 5A, bottom left). For slower evolutionary rates, our results match the predictions from our theory that these equilibria will be unstable (fig. $5 C$ ). In figure 5A, we show the critical heritability at which this change in stability occurs (grayscale shading at bottom left). An equilibrium that is more evolutionarily stable (smaller $d$ ) has a lower critical heritability that changes stability (vertical transects at bottom left of fig. 5A).

\section{Competition Model II: Eco-Evolutionary Feedbacks Are Destabilizing}

Different traits may lead to different relationships between demography and selection, thereby affecting the role of ecoevolutionary feedbacks on stability. Suppose now that the evolving competitor (species 1) has a trait that increases the antagonistic interactions between species 1 and 2, while decreasing intraspecific antagonism (fig. 4II). An example of such a trait is when time spent on antagonistic interactions with interspecific competitors reduces time spent on antagonistic interactions with intraspecific competitors. More specifically, in territorial organisms, competition with heterospecifics for space can reduce individual range overlap with conspecifics (Hoi et al. 1991).

There are two key differences from traits in model I: increased density of species 1 selects for higher trait values, while increases in species 2 select for lower trait values. These differences are captured in the signs of vectors $b$ and $c$ :

$$
b=\left[\begin{array}{c}
0 \\
-
\end{array}\right], \quad c=[+-] .
$$

The feedback term for slow evolution (evo-eco-evo pathway) is

$$
\begin{aligned}
& c A^{-1}(-b)=\left[\begin{array}{ll}
+ & -
\end{array}\right]\left[\begin{array}{ll}
- & + \\
+ & -
\end{array}\right]\left[\begin{array}{l}
0 \\
+
\end{array}\right]=[+] . \\
& \Delta N=\left[\begin{array}{l}
+ \\
-
\end{array}\right]
\end{aligned}
$$

The first step of this pathway is the same as in model (1): a small increase in the trait leads to an increase in the density of species 1 and decrease of species 2 (from $\Delta N$ ). However, these population density changes result in a selective pressure to further increase the trait values, which magnifies the perturbation. This creates a positive feedback, which has destabilizing effects (top half of fig. $2 B$ ). Thus, even if the equilibrium is ecologically and evolutionarily stable, it can be overall unstable due to these destabilizing evo-eco-evo feedbacks (iii in fig. $2 B, 2 C$ ). 

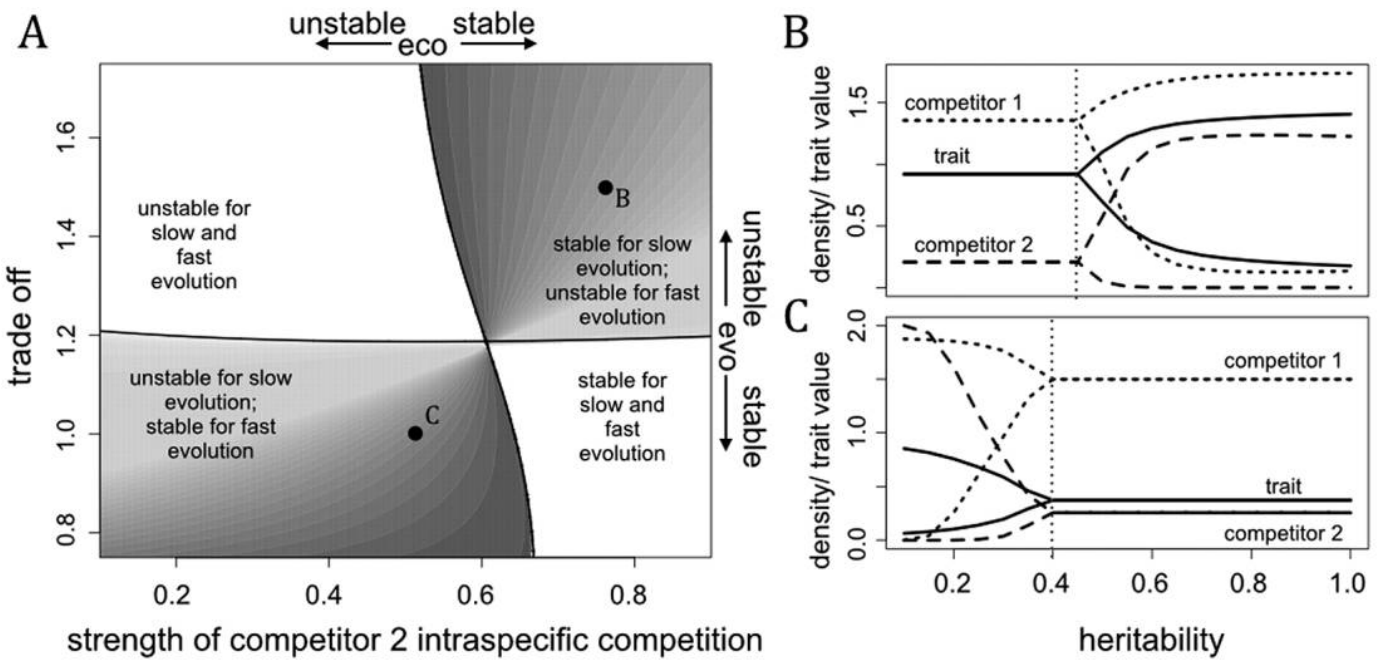

Figure 5: Ecological stability, evolutionary stability, and overall stability for competing species in model I. In $A$, the $X$-axis is the strength of intraspecific competition of the nonevolving species and the $Y$-axis is the strength of the trade-off between intra- and interspecific competition. The vertical and horizontal black curves correspond to $s(A)=0$ and $d=0$, separating ecological stability and evolutionary stability, respectively. Each resulting region is labeled by whether the equilibria are overall stable for slow and fast evolution. At the top right and bottom left, there is a switch in stability at a critical rate of evolution. The grayscale shading corresponds to when the switch in stability occurs, with dark gray representing the slowest critical rate and light gray representing the fastest. $B, C$, Long-term minimum and maximum densities and trait values for varying heritabilities, which determine the relative rate of evolution. The vertical dotted line corresponds to the critical rate, and the parameters correspond to points $\mathrm{B}$ and $\mathrm{C}$ in $\mathrm{A}$.

When evolution is fast, the critical eco-evolutionary feedback is via the eco-evo-eco pathway given by

$$
b d^{-1}(-c)=\left[\begin{array}{cc}
0 & 0 \\
- & +
\end{array}\right],
$$

which is destabilizing because adding $b d^{-1}(-c)$ to $A$ decreases the determinant and increases the trace.

To summarize, the important eco-evolutionary feedbacks for slow and fast evolution are both destabilizing, which is the opposite of that found for traits in model I. We tested this general theory with a model modified from Vasseur et al. (2011) to reflect traits of this type (see app. C). Consistent with our theory, we find that eco-evolutionary feedbacks are often sufficiently destabilizing such that equilibria with stable ecological and evolutionary subsystems are overall unstable (app. C).

\section{Effects of Correlated Multitrait Evolution on Stability}

Previous work has shown that in purely evolutionary models, when a single species is evolving in multiple quantitative traits that contribute independently to fitness, correlations between these traits will not qualitatively affect evolutionary stability (Lande 1979). Correlations can, however, affect the degree of stability and slow down or speed up the return to a stable equilibrium, following a perturbation. Here, we ask what is the role of correlations on stability in light of eco-evolutionary feedbacks.
First, we ask whether correlations can qualitatively change stability when multiple quantitative traits of a single species within the community evolve (using eq. [3]). For simplicity, we assume that all the traits have equal genetic variances so that the genetic covariance in equation (3) can be written as $v_{m j}=v \rho_{m j}$, where $v$ is the constant genetic variance and $\rho_{m j}$ is the genetic correlation between traits $m$ and $j$. Then, if we let $\varepsilon=v$, we can express matrices $C$ and $D$ in the Jacobian (4) as $P \tilde{C}$ and $P \tilde{D}$, where $P$ is the genetic correlation matrix with elements $\rho_{m j}$,

$$
\tilde{C}=\left[\begin{array}{ccc}
\frac{\partial^{2} f}{\partial N_{1} \partial x_{1}} & \cdots & \frac{\partial^{2} f}{\partial N_{k} \partial x_{1}} \\
\vdots & & \vdots \\
\frac{\partial^{2} f}{\partial N_{1} \partial x_{\ell}} & \cdots & \frac{\partial^{2} f}{\partial N_{k} \partial x_{\ell}}
\end{array}\right]
$$

and $\tilde{D}$ is a diagonal matrix with elements $\partial^{2} f / \partial x_{j}^{2}$, where $f$ is the fitness of the evolving species. When evolution is slow, the conditions for overall stability are ecological stability $(s(A)<$ 0 ; unaffected by correlations) and $s\left(D+C A^{-1}(-B)\right)=$ $s\left(P\left(\tilde{D}+\tilde{C} A^{-1}(-B)\right)\right)<0$. As the correlation matrix $P$ can transform the evo-eco-evo feedback, correlations can change stability. On the other hand, when evolution is fast, the conditions for overall stability are evolutionary stability $(s(D)=$ $s(P \tilde{D})<0)$ and $s\left(A+B D^{-1}(-C)\right)=s\left(A+B \tilde{D}^{-1}(-\tilde{C})\right)<0$. 
As Lande (1979) has shown, the sign of $s(P \tilde{D})$ is unaffected by correlation matrix $P$, and correlations between multiple traits do not qualitatively affect stability when evolution is sufficiently fast.

To test for the effects of correlations on stability with ecoevolutionary feedbacks, we apply our results to a three-species Lotka-Volterra food chain model, in which two traits of the herbivore evolve, one that determines its ability to consume the basal resource species and another that influences its defense against the top predator. We assume that there is a trade-off between resource consumption and intraspecific competition and a trade-off between defense and intraspecific competition. For example, in many territorial birds, larger territory sizes increase resource consumption but also intraspecific competition (Davies and Houston 1981), while nesting behavior, a trait potentially correlated to territory size, affects both intraspecific competition as well as protection against predators (Martin and Li 1992; Cockle et al. 2010).

To model these trade-offs, we use modified versions of the functions from Schreiber et al. (2011) and Vasseur et al. (2011). While we assume that these traits act independently to determine the interaction strengths with the basal species and top predator, we allow for the two traits to be genetically correlated (see app. D for more details). While these model assumptions determine the sign structure of matrices $A, B$, and $\tilde{C}$, this sign structure is not enough to indicate whether the eco-evo-eco nor the evo-eco-evo feedback is stabilizing or destabilizing (app. D). Consequently, we explore this model numerically.

We plot contours of the stability modulus of the Jacobian (4) for varying genetic variances (evolutionary rates) and correlations between the two traits (fig. 6A) on equilibria that are ecologically stable and evolutionarily unstable. First, we find that when evolution is slow, evo-eco-evo feedbacks are sufficient to stabilize the equilibrium for all correlations (fig. 6A). Second, we find a U-shaped relationship between the effect of the genetic variance and correlation on stability (fig. 6A). In particular, for intermediate rates of evolution, correlations qualitatively change stability: only when the two traits are sufficiently positively or negatively correlated is the equilibrium overall stable (fig. $6 B$ ). Finally, for sufficiently fast evolution, the equilibrium is unstable for all appropriate correlation values.

\section{Discussion \\ Implications of Our Results}

Existing empirical and theoretical studies have demonstrated that eco-evolutionary feedbacks can have substantial effects on community stability, but this has largely focused on specific ecological modules (Abrams and Matsuda 1997; Becks et al. 2010; Cortez and Ellner 2010; Schreiber et al. 2011; Vasseur et al. 2011; Steiner and Masse 2013; Patel and Schreiber 2015; Cortez 2016). Our analysis unifies these existing stud-
A

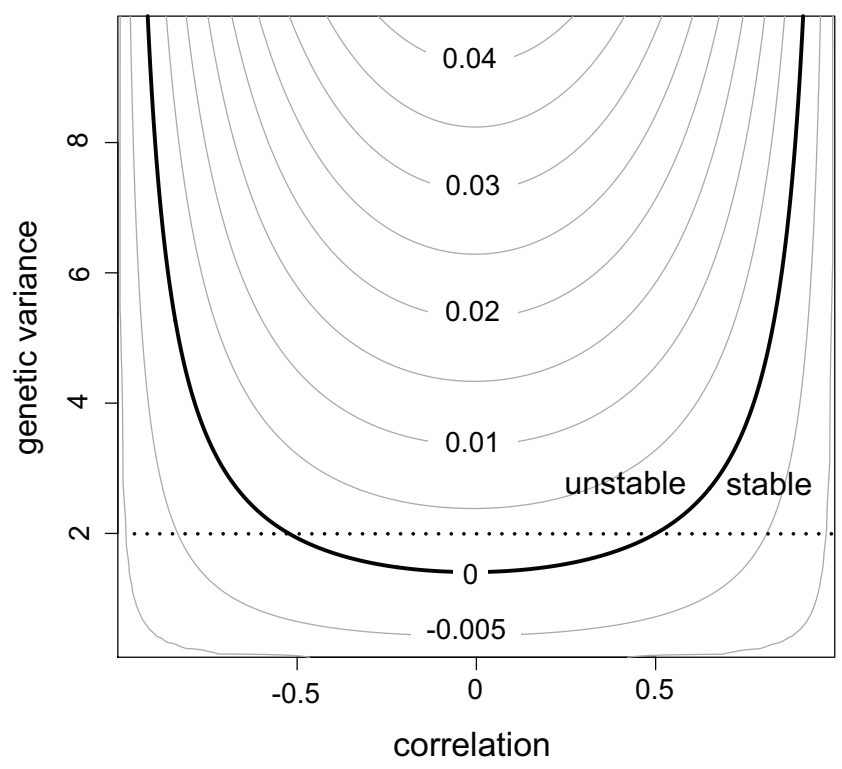

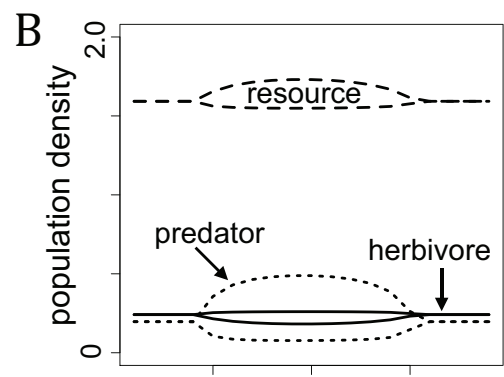

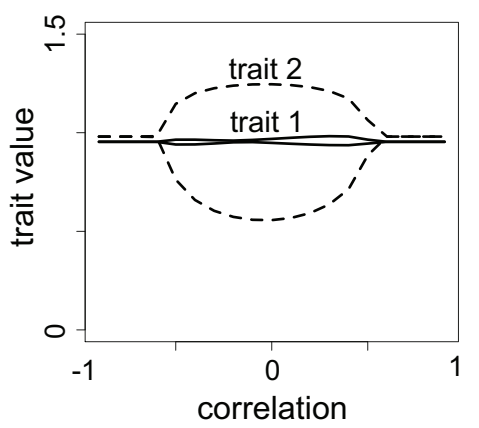

Figure 6: Effects of trait correlations on stability of food chains. A, Contours of the stability modulus for varying genetic variances (rates of evolution) and correlations. $B$, Minimum and maximum population densities and trait values at the eco-evolutionary attractor for a fixed rate of evolution (corresponding to the horizontal dotted line in $A$ ) and varying correlations. 
ies and provides a general theory for mechanistically understanding how eco-evolutionary feedbacks impact stability, which can be applied to model communities with any number of species and evolving traits. In particular, we provide conditions for stability for when evolution is fast and slow relative to ecology. By interpreting these conditions, we draw inferences on when and how direct ecological, direct evolutionary, and indirect eco-evolutionary feedbacks impact stability and how these impacts depend on the relative timescales of the ecological and evolutionary processes.

Recently, Hendry (2016) highlighted that understanding the effects of the relative rate of evolutionary processes to ecological processes and the role of direct and indirect effects on community stability is a fundamental problem in ecoevolutionary dynamics. Our work makes several advances on this problem. The conditions for stability when evolution is slow relative to ecology are that the direct ecological effects must be stable and the sum of the direct evolutionary effects with the indirect evo-eco-evo feedbacks must be stable. In contrast, when evolution is fast, the direct evolutionary effects must be stable and the sum of the direct ecological effects with the indirect eco-evo-eco feedbacks must be stable. As we describe in the main text, these results have a graphical interpretation as a two-step response to perturbations in systems with a separation of timescales. Importantly, these conditions highlight that, on one hand, eco-evolutionary feedbacks can destabilize an equilibrium that is ecologically and evolutionarily stable and, on the other, can stabilize an equilibrium that is unstable in the slower dynamic.

Our theory indicates that an equilibrium may not be stable for all evolutionary timescales; that is, system stability can depend on the relative rate of evolution to ecology. In particular, it may be stable for slow evolution and unstable with fast evolution, or vice versa. We can draw two general conclusions that are true for all types of species interactions in this framework. First, if an equilibrium is evolutionarily unstable but stabilized by the slow evo-eco-evo feedback, then the equilibrium will destabilize when evolution occurs sufficiently quickly. For example, in the first model of traits for two competing species, this destabilization occurs despite the eco-evolutionary feedbacks having a stabilizing effect for slow and fast evolution (fig. 5). Second, if an equilibrium is ecologically unstable but stabilized by the fast eco-evo-eco feedback, then it will lose its stability with slow evolution. These stability changes for varying rates of evolution have been observed theoretically in predator-prey (Abrams and Matsuda 1997; Cortez 2016), competition (Vasseur et al. 2011), and intraguild predation communities (Patel and Schreiber 2015) as well as suggested experimentally in predator-prey communities (Becks et al. 2010). Our general results suggest that one cause for the loss or gain of stability with varying rates of evolution observed in these studies may be the switch in the dynamics that dominates the fast response.
Our three examples show that the main results can be applied to communities with different types of species interactions and evolutionary constraints. Applying our results to two-species competition, we demonstrate how qualitative information about the relationships between traits and population growth sometimes is sufficient to infer the effects of eco-evolutionary feedbacks on stability. Importantly, qualitative differences in these relationships can alter whether the feedback is stabilizing or destabilizing. For a trait that increases the competitive ability of the evolving species against heterospecifics at the cost of increasing competition among conspecifics (competition model I), eco-evolutionary feedbacks are stabilizing. In contrast, when the trait increases interference competition between heterospecifics (competition model II) while reducing competition among conspecifics, eco-evolutionary feedbacks are destabilizing. In both cases, in the evo-eco-evo feedback, a small positive perturbation in the trait leads to an increase and decrease in the density of species 1 and 2, respectively. In the first case, these population density changes drive a selection pressure that opposes the perturbation. In the second case, these population density changes drive selection pressures that amplify the initial trait perturbation.

When a single species evolves in multiple quantitative traits, classic evolutionary theory asserts that correlations cannot qualitatively impact evolutionary stability (Lande 1979). These correlations can, however, alter the trajectories of how traits approach (i.e., stabilizing selection) or diverge (e.g., disruptive selection) from an equilibrium. When evolution is sufficiently fast relative to ecology, traits evolve very quickly to the equilibrium relative to the ecological process and so the trajectories traits take to get to the equilibrium (and, hence, correlations) have little effect on the overall eco-evolutionary feedbacks. However, as the relative rate of evolution becomes more commensurate or slower than the ecological dynamics, the traits no longer reach their equilibrium before eliciting an ecological response. Hence, the correlation effects on trait trajectories become important in the eco-evolutionary feedbacks. Importantly, in this way, stability depends critically on correlations when evolution occurs at slow or intermediate rates relative to ecology.

\section{Comments on Our Framework}

We illustrated our results with Lande's quantitative genetics framework (Lande 1976). Our results are also applicable to two additional notable existing frameworks of modeling evolutionary dynamics: the explicit multilocus evolutionary framework and the adaptive dynamics framework. Hence, our results can be applied to many distinct descriptions of the evolutionary process.

The multilocus modeling framework describes the evolutionary process by characterizing fitness differences among 
explicit genotypes in the population and tracking genotypic frequencies as the evolutionary variable (Doebeli 1997; Yamaguchi et al. 2011; Yamamichi and Ellner 2016). In this framework, the genotypic frequencies are the populationlevel quantitative values of interest. When fitness differences between genotypes are small, evolution may occur slowly relative to ecological population density changes. On the other hand, if population growth is constrained, then ecological dynamics may occur slowly relative to evolutionary genotype frequency changes.

In the adaptive dynamics framework, populations are assumed to be monomorphic and clonal, with evolution occurring due to mutations of small effect. Since it is assumed that mutations are rare, the evolutionary dynamics are assumed to be much slower than the ecological dynamics. Hence, our mathematical results for slow evolution can also be applied to an adaptive dynamics framework for studying ecoevolutionary dynamics. In appendix E, we describe in more detail the interpretations of the stability conditions in an adaptive dynamics framework.

Finally, timescale separations may also occur among or within behavioral, ecological, or ecosystem processes relevant to the system dynamics. For all of these scenarios, the stability conditions presented here can still be applied. For example, our conditions can be used to understand stability in models with plastic or behaviorally optimizing traits, which are often thought to change very quickly relative to ecological processes (e.g., Abrams 1992; Kondoh 2003; Ma et al. 2003), to purely ecological models, in which a subset of species naturally reproduces and dies at a faster rate than another set of species (e.g., Muratori and Rinaldi 1992), or to combinations of these cases, as in Takimoto et al. (2009). In particular, the results can be applied to eco-evolutionary models with mixed timescale separation, such as when some subset of species evolves and reproduces more quickly than the remaining set. Importantly, the responses of a system to perturbations may depend on which subset of processes occurs slowly relative to the remaining set of processes (Takimoto et al. 2009).

\section{Future Directions}

Our analysis shows that for sufficiently slow evolutionary rates, the evo-eco-evo feedback is critical, while for sufficiently fast evolutionary rates, the eco-evo-eco feedback is critical. What does this mean for intermediate rates of evolution? Except for the special case in which the slow dynamics are onedimensional (see app. B), we cannot in general make stability conclusions for intermediate rates of evolution based on our understanding of fast and slow evolution. For intermediate rates of evolution, additional eco-evolutionary interactions not accounted for in the feedback pathways given for slow and fast evolution will play a role and can alter stability. In particular, even if an equilibrium is stable for slow and fast evolution, it may be unstable for intermediate rates of evolution (e.g., see Cortez 2016; Cortez and Patel 2017).

Simple and general analytical results for partitioning ecoevolutionary feedback effects for intermediate rates are limited. A related approach to stability that may be enlightening for intermediate rates of evolution is loop analysis, which partitions stability conditions in terms of feedback loops of varying length (Levins 1968). By identifying which conditions are not met, one may be able to pinpoint which loop or set of loops are responsible for the loss of stability (for a recent example in a pure ecological model, see Neutel and Thorne 2014). The obstacle of this approach is that the number of loops to consider grows rapidly with the number of species and traits. Nonetheless, further development in this area may help to understand the role of eco-evolutionary feedbacks on stability and bridge the slow and fast limits.

Through our competition examples, we showed that purely qualitative information about the relationships between traits and population growth (signs of the matrices) were sufficient for predicting whether eco-evolutionary feedbacks were stabilizing or destabilizing. In most cases of more complex models, sign structure alone will not be sufficient to infer the effects of eco-evolutionary feedbacks, as it is in the two-species competition cases considered here. This is consistent with the pattern May (1973) noted when he used sign structure of purely ecological community matrices to determine stability. Hence, for more complex models, our theory is still applicable when we can obtain quantitative information about these eco-evolutionary relationships, that is, the Jacobian elements.

One way in which we can obtain this information is to use empirical time series data to parameterize a model and then estimate the Jacobian (Deyle et al. 2016). From this, we can observe whether there is an evident timescale separation and, if so, apply our method to partition out the influence of pure ecology, pure evolution, and eco-evolutionary feedbacks on stability. Alternatively, if the timescales of ecology and evolution are similar, then we can ask how stability would be different if one process was sped up or slowed down to occur much more quickly than the other. By applying these methods to empirical systems, we might be able to identify the relative contributions of ecology, evolution, and eco-evolutionary feedbacks on community stability or instability.

Finally, in this work, we focus particularly on the effects of eco-evolutionary feedbacks on community stability, which determines how a community at equilibrium will respond to small yet potentially frequent perturbations (Schreiber 2006). While stability is a fundamental property of communities, there are many other properties of interest to community ecologists, including the persistence of species, resilience to large perturbations, and robustness (Hutson and Schmitt 1992; Schreiber 2000; Meszéna et al. 2006; Barabás et al. 2012; Barabás and D'Andrea 2016; Klauschies et al. 2016). Develop- 
ing a general framework for identifying the effects of feedbacks on these and other critical properties of communities is an important avenue for future research to build a broader understanding of how communities will respond in the face of new ecological and evolutionary pressures.

\section{Acknowledgments}

We thank Thomas Schoener, Jaime Ashander, Axel Saenz, Casey terHorst, Peter Zee, Kelsey Lyberger, Sam Fleischer, Mathew Leibold, and two anonymous reviewers for useful discussion on this topic and comments on the manuscript. Financial support by the National Science Foundation (DMS1313418) to S.J.S., the American Association of University Women Dissertation Fellowship to S.P., and the Austrian Science Fund (FWF; P25188-N25) to Reinhard Burger at the University of Vienna is gratefully acknowledged.

\section{Literature Cited}

Abrams, P. A. 1992. Adaptive foraging by predators as a cause of predator-prey cycles. Evolutionary Ecology 6:56-72.

Abrams, P. A., and H. Matsuda. 1997. Prey adaptation as a cause of predator-prey cycles. Evolution 51:1742-1750.

Alberti, M. 2015. Eco-evolutionary dynamics in an urbanizing planet. Trends in Ecology and Evolution 30:114-126.

Barabás, G., and R. D’Andrea. 2016. The effect of intraspecific variation and heritability on community pattern and robustness. Ecology Letters 19:977-986.

Barabás, G., S. Pigolotti, M. Gyllenberg, U. Dieckmann, and G. Meszéna. 2012. Continuous coexistence or discrete species? a new review of an old question. Evolutionary Ecology Research 14:523-554.

Becks, L., S. P. Ellner, L. Jones, and N. Hairston Jr. 2010. Reduction of adaptive genetic diversity radically alters eco-evolutionary community dynamics. Ecology Letters 13:989-997.

Bender, E., T. Case, and M. Gilpin. 1984. Perturbation experiments in community ecology: theory and practice. Ecology 65:1-13.

Cockle, K., K. Martin, and M. Drever. 2010. Supply of tree-holes limits nest density of cavity-nesting birds in primary and logged subtropical Atlantic forest. Biological Conservation 143:2851-2857.

Cortez, M. H. 2015. Coevolution-driven predator-prey cycles: predicting the characteristics of eco-coevolutionary cycles using fast-slow dynamical systems theory. Theoretical Ecology 8:369-382.

- 2016. How the magnitude of prey genetic variation alters predator-prey eco-evolutionary dynamics. American Naturalist 188: 329-341.

Cortez, M. H., and S. P. Ellner. 2010. Understanding rapid evolution in predator-prey interactions using the theory of fast-slow dynamical systems. American Naturalist 176:E109-E127.

Cortez, M. H., and S. Patel. 2017. The effects of predator evolution and genetic variation on predator-prey population-level dynamics. Bulletin of Mathematical Biology 79:1510-1538.

Cortez, M. H., and J. S. Weitz. 2014. Coevolution can reverse predatorprey cycles? Proceedings of the National Academv of Sciences of the USA 111:7486-7491.

Darimont, C., S. Carlson, M. Kinnison, P. Paquet, T. Reimchen, and C. Wilmers. 2009. Human predators outpace other agents of trait change in the wild. Proceedings of the National Academy of Sciences of the USA 106:952-954.

Davies, N., and A. Houston. 1981. Owners and satellites: the economics of territory defence in the pied wagtail, Motacilla alba. $\underline{\text { Journal of }}$ Animal Ecology 50:157-180.

DeLong, J. P., V. E. Forbes, N. Galic, J. P. Gibert, R. G. Laport, J. S. Phillips, and J. M. Vavra. 2016. How fast is fast? eco-evolutionary dynamics and rates of change in populations and phenotypes. Ecology and Evolution 6:573-581.

Deyle, E., R. May, S. Munch, and G. Sugihara. 2016. Tracking and forecasting ecosystem interactions in real time. Proceedings of the Roval Societv B 283:20152258.

Doebeli, M. 1997. Genetic variation and persistence of predator-prey interactions in the Nicholson-Bailey model. Lournal of Theoretical Biology 188:109-120.

Fussmann, G., M. Loreau, and P. A. Abrams. 2007. Eco-evolutionary dynamics of communities and ecosystems. Functional Ecology 21: 465-477.

Gavrilets, S. 1997. Coevolutionary chase in exploiter-victim systems with polygenic characters. Journal of Theoretical Biology 186:527-534.

Geritz, S. A., E. Kisdi, G. Meszéna, and J. A. Metz. 1998. Evolutionarily singular strategies and the adaptive growth and branching of the evolutionary tree. Evolutionary Ecology 12:35-57.

Hendry, A. P. 2013. Key questions in the genetics and genomics of eco-evolutionary dynamics. Heredity 111:456-466.

. 2016. Eco-evolutionary dynamics. Princeton University Press, Princeton, NJ.

Hendry, A. P., M. Kinnison, M. Heino, T. Day, T. Smith, G. Fitt, C. Bergstrom, J. Oakeshott, P. Jørgensen, and M. Zalucki. 2011. Evolutionary principles and their practical application. Evolutionary Applications 4:159-183.

Hoi, H., T. Eichler, and J. Dittami. 1991. Territorial spacing and interspecific competition in three species of reed warblers. Oecologia 87:443-448.

Hutson, V., and K. Schmitt. 1992. Permanence and the dynamics of biological systems. Mathematical Biosciences 111:1-71.

Kasada, M., M. Yamamichi, and T. Yoshida. 2014. Form of an evolutionary tradeoff affects eco-evolutionary dynamics in a predator-prey system. Proceedings of the National Academv of Sciences of the USA 111:16035-16040.

Klauschies, T., D. A. Vasseur, and U. Gaedke. 2016. Trait adaptation promotes species coexistence in diverse predator and prey communities. Ecology and Evolution 6:4141-4159.

Kondoh, M. 2003. Foraging adaptation and the relationship between food-web complexity and stability. Science 299:1388-1391.

Kopp, M., and S. Matuszewski. 2013. Rapid evolution of quantitative traits: theoretical perspectives. Evolutionary Applications 7:169-191.

Lande, R. 1976. Natural selection and random genetic drift in phenotypic evolution. Evolution 30:314-334.

1979. Quantitative genetic analysis of multivariate evolution, applied to brain: body size allometry. Evolution 33:402-416.

Lande, R., and S. J. Arnold. 1983. The measurement of selection on correlated characters. Evolution 37:1210-1226.

Lankau, R. A., P. S. Jorgensen, D. J. Harris, and A. Sih. 2011. Incorporating evolutionary principles into environmental management and policy. Evolutionary Applications 4:315-325.

Levins, R. 1968. Evolution in changing environments: some theoretical explorations. Princeton University Press, Princeton, NJ.

Ma, B., P. Abrams, and C. Brassil. 2003. Dynamic versus instantaneous models of diet choice. American Naturalist 162:668-684. 
Marrow, P., U. Dieckmann, and R. Law. 1996. Evolutionary dynamics of predator-prey systems: an ecological perspective. Journal of Mathematical Biology 34:556-578.

Martin, T., and P. Li. 1992. Life history traits of open- vs. cavity-nesting birds. Ecology 73:579-592.

May, R. M. 1973. Qualitative stability in model ecosystems. Ecology 54:638-641.

Meszéna, G., M. Gyllenberg, L. Pásztor, and J. A. Metz. 2006. Competitive exclusion and limiting similarity: a unified theory. Theoretical Population Biology 69:68-87.

Muratori, S., and S. Rinaldi. 1992. Low- and high-frequency oscillations in three-dimensional food chain systems. SIAM Journal on Applied Mathematics 52:1688-1706.

Neutel, A. M., and M. A. Thorne. 2014. Interaction strengths in balanced carbon cycles and the absence of a relation between ecosystem complexity and stability. Ecology Letters 17:651-661.

Nuismer, S., and M. Doebeli. 2004. Genetic correlations and the coevolutionary dynamics of three-species systems. Evolution 58:11651177.

Patel, S., and S. J. Schreiber. 2015. Evolutionarily driven shifts in communities with intraguild predation. American Naturalist 186:E98E110.

Pimentel, D., W. Nagel, and J. L. Madden. 1963. Space-time structure of the environment and the survival of parasite-host systems. American Naturalist 97:141-167.

Pimm, S., and J. Lawton. 1978. On feeding on more than one trophic level. Nature 275:542-544.

Pruitt, J. N., and S. E. Riechert. 2009. Frequency-dependent success of cheaters during foraging bouts might limit their spread within colonies of a socially polymorphic spider. Evolution 63:2966-2973.

Reznick, D. N. 2013. A critical look at reciprocity in ecology and evolution: introduction to the symposium. American Naturalist 181(suppl.): S1-S8.

Sanchez, A., and J. Gore. 2013. Feedback between population and evolutionary dynamics determines the fate of social microbial populations. PLoS Biology 11:e1001547.

Schreiber, S. J. 2000. Criteria for $C^{\mathrm{r}}$ robust permanence. Journal of Differential Equations 162:400-426.

- 2006. Persistence despite perturbations for interacting populations. Iournal of Theoretical Biology 242:844-852.

Schreiber, S. J., R. Bürger, and D. I. Bolnick. 2011. The community effects of phenotypic and genetic variation within a predator population. Ecology 92:1582-1593.

Schreiber, S. J., and S. Patel. 2015. Evolutionarily induced alternative states and coexistence in systems with apparent competition. Natural Resource Modelling 28:475-496.
Seger, J. 1988. Dynamics of some simple host-parasite models with more than two genotypes in each species. Philosophical Transactions of the Royal Society B 319:541-555.

Steiner, C., and J. Masse. 2013. The stabilizing effects of genetic diversity on predator-prey dynamics. F1000Research 2013:43.

Takimoto, G., T. Iwata, and M. Murakami. 2009. Timescale hierarchy determines the indirect effects of fluctuating subsidy inputs on in situ resources. American Naturalist 173:200-211.

terHorst, C. 2010. Evolution in response to direct and indirect ecological effects in pitcher plant inquiline communities. American Naturalist 176:675-685.

Tilman, D. 2007. Interspecific competition and multi species coexistence. Pages 85-97 in R. May and A. McLean, eds. Theoretical ecology: principles and applications. Oxford University Press, New York.

Turcotte, M. M., D. N. Reznick, and J. Hare. 2011. The impact of rapid evolution on population dynamics in the wild: experimental test of eco-evolutionary dynamics. Ecology Letters 14:1084-1092.

Vasseur, D. A., P. Amarasekare, V. H. Rudolf, and J. M. Levine. 2011. Eco-evolutionary dynamics enable coexistence via neighbor-dependent selection. American Naturalist 178:E96-E109.

Yamaguchi, W., M. Kondoh, and M. Kawata. 2011. Effects of evolutionary changes in prey use on the relationship between food web complexity and stability. Population Ecology 53:59-72.

Yamamichi, M., and S. Ellner. 2016. Antagonistic coevolution between quantitative and Mendelian traits. Proceedings of the Roval Society B 283:20152926.

Yodzis, P. 1988. The indeterminacy of ecological interactions as perceived through perturbation experiments. Ecology 69:508-515.

Yoshida, T., L. Jones, S. P. Ellner, G. Fussmann, and N. G. Hairston Jr. 2003. Rapid evolution drives ecological dynamics in a predator-prey system. Nature 424:303-306.

\section{References Cited Only in the Online Appendixes}

Hofbauer, J., and K. Sigmund. 1998. Evolutionary games and population dynamics. Cambridge University Press, Cambridge.

R Core Development Team. 2013. R: a language and environment for statistical computing. R Foundation for Statistical Computing, Vienna.

Soetaert, K. 2009. rootSolve: nonlinear root finding, equilibrium and steady-state analysis of ordinary differential equations. R package, version 1.6.

Soetaert, K., T. Petzoldt, and R. W. Setzer. 2010. Solving differential equations in R: package deSolve. Journal of Statistical Software 33: 1-25.

Symposium Editor: Mathew A. Leibold 